\title{
Are we repeating mistakes of the past? A review of the evidence for esketamine - CORRIGENDUM
}

\author{
Mark A. Horowitz and Joanna Moncrieff
}

\section{Keywords}

Esketamine; ketamine; depression; antidepressants; suicide; corrigendum.

\section{Copyright and usage}

(c) The Author(s), 2021. Published by Cambridge University Press on behalf of the Royal College of Psychiatrists. https://doi.org/10.1192/bjp.2020.89, Published online by Cambridge University Press, $27^{\text {th }}$ May 2020

It should be emphasised that Table 1 in the Analysis ${ }^{1}$ combines data from Phase 2 trials (including open-label and placebo-controlled trial periods) and Phase 3 trials (placebo-controlled efficacy studies, a maintenance of effect study and a long-term open label safety study) submitted by Janssen to the FDA (as indicated by superscript ' $b$ ' in the Table), therefore the data does not represent a randomised comparison, and definitive causal inferences cannot be drawn. As indicated by the denominators for each entry there were more patients exposed to esketamine than placebo (and for longer periods) which may be one reason for the higher numbers of suicides in the esketamine group. However, it is worth noting other data that suggest that increased suicidality may be a feature of esketamine treatment. In the long-term safety study $14.5 \%$ of patients on esketamine (in a population selected for their lack of active suicidality) reported 'treatment-emergent' suicidal ideation (114/784), 6 patients attempted suicide in addition to the one completed suicide. ${ }^{2}$ Furthermore, a recent analysis of post-marketing surveillance data reported to the FDA for the 12 months since esketamine was licensed in the US there have already been 64 reports of suicidal ideation, 11 completed suicides, and 6 attempted suicides attributed to esketamine. ${ }^{3}$ This represents a 24 -fold increased risk of report for suicidal ideation for esketamine compared with other drugs, and a 6-fold increased risk for completed suicide. ${ }^{3}$ The authors of this paper concluded that the safety of esketamine required "urgent clarification."

It should also be clarified that although ketamine is not used routinely as an anaesthetic agent in high-income countries, like the UK, because of its unfavourable balance of risks and harms, it is used more frequently in low-income countries.

Mark A. Horowitz; Joanna Moncrieff

\section{References}

1 Horowitz MA, Moncrieff J. Are we repeating mistakes of the past? A review of the evidence for esketamine. Br J Psychiatry 2020; 1-4. https://doi.org/10. 1192/bjp.2020.89.

2 Wajs E, Aluisio L, Holder R, et al. Esketamine Nasal Spray Plus Oral Antidepressant in Patients With Treatment-Resistant Depression. J Clin Psychiatry 2020; 81: 4-5.

3 Gastaldon C, Raschi E, Kane JM, Barbui C, Schoretsanitis G. Post-Marketing Safety Concerns with Esketamine: A Disproportionality Analysis of Spontaneous Reports Submitted to the FDA Adverse Event Reporting System. Psychother Psychosom 2020; 1-8. 